\title{
Communication
}

\section{Screening of Aptamers That Bind to the Multivalent Aminoglycoside Amikacin}

\author{
AHM Khurshid Alam ${ }^{1,3}$, Yoshiko Miura ${ }^{2}$ and Toshifumi Tsukahara ${ }^{3 *}$
}

1 Department of Pharmacy, University of Rajshahi, Rajshahi-6205, Bangladesh;khurshid.jaist@gmail.com

2 Department of Chemical Systems and Engineering, Graduate School of Engineering, Kyshu University, 744 Motooka, Nishi-ku, Fukuoka-819-0395, Japan; miuray@chem-eng.kyushu-u.ac.jp

3 School of Materials Science, Japan Advanced Institute of Science and Technology, 1-1 Asahidai, Nomi City, Ishikawa-923-1292, Japan; tukahara@jaist.ac.jp

* Correspondence: E-mail: tukahara@jaist.ac.jp; Tel.: +81-761-51-1640; Fax.: +81-761-51-1149

\begin{abstract}
Increased awareness of the multiple roles of RNA molecules has led to the realization that, in addition to their structural and functional roles, RNAs can be drug targets for small molecular therapy. The aim of this study was to identify multivalent amikacin specific RNA aptamers that can be a new target sites for aminoglycoside antibiotics, including amikacin using the systemic evolution of ligands by exponential enrichment (SELEX) method. Amikacin, a member of the aminoglycoside group of antibiotics, binds to

specific sites in bacterial 16S ribosomal RNAs (rRNAs) and interferes with protein synthesis, leading to cell death. Here, we used the SELEX method to isolate high affinity RNA fragments (aptamers) that bind to amikacin. After five rounds of SELEX selection, in which a linear N25 DNA template was used for the first selection cycle, the resulting RNA was cloned and sequenced. Among the 38 clones generated, five groups of sequences (groups A through E) containing nine conserved motifs were identified. The sequences of groups A and B were almost identical, indicating that the selected RNA was enriched. Subsequently, the Basic Local Alignment Search Tool program was used to search for the conserved motifs in bacterial 16S rRNA sequences. Strikingly, no sequence homology was observed, suggesting that the conserved sequences (motifs) identified in this study may be novel target sites for amikacin.
\end{abstract}

Keywords: SELEX; aptamer; amikacin

\section{Introduction}

Aminoglycosides (AGs) are a class of antibiotics that contain a characteristic aminocyclitol ring, usually streptidine or 2-deoxystreptamine, and two or more amino sugars joined in a glycosidic linkage to a hexose nucleus [1,2]. Characterizing the structure of AGs is important to understand their chemical and biological activities. AGs are basic and strongly polar compounds that are positively charged, which contributes to their antimicrobial activity [3]. Because they are polycationic, AGs show a binding affinity for nucleic acids (RNA and DNA); specifically, they possess high affinities for certain portions of negatively charged RNAs, particularly the A-site region on the $16 \mathrm{~S}$ subunit of prokaryotic ribosomal RNA (rRNA) [4]. Thus, AGs exert their antibacterial effects by interfering with ribosomal function, which ultimately results in disruption of protein synthesis.

The most commonly used AGs in hospitals are amikacin, gentamicin, and tobramycin [5]. Amikacin is a semi-synthetic AG antibiotic derived by acetylation of kanamycin A; it is particularly effective against bacteria that are resistant to other AGs because its chemical structure renders it less susceptible to inactivating enzymes [6]. Like other AGs, amikacin irreversibly binds to a specific A-site on the 16S rRNA subunit of the $30 \mathrm{~S}$ ribosome to prevent the formation of an initiation complex with mRNA, thereby inhibiting protein synthesis [7]. More specifically, amikacin binds to four nucleotides of $16 \mathrm{~S}$ 
rRNA and a single amino acid of protein S12 [8]. Despite its wide ranging antibacterial effects, resistance to amikacin can be achieved via various mechanisms, including the production of amikacin-modified enzymes (acetyltransferase, nucleotidyltransferase, and phosphotransferase), the reduction of antibiotic penetration of the outer membrane protein, the acquisition of reduced affinity by changing nucleotides within the $16 \mathrm{~S}$ rRNA, and augmented excretion by an efflux pump system $[3,9,10]$. To overcome these problems, it is highly desirable to synthesize a modified amikacin molecule that has a higher RNA-binding affinity, better selectivity and antibacterial activity, and stronger resistance to AG-modifying enzymes than the parent molecule. Recently, Miura and her colleagues' synthesized polymeric amikacin (multivalent amikacin) by chemoenzymatic esterification of the parent structure [11]. Chemoselective reactions are important for the modification of AGs because they possess multiple amino and hydroxyl groups. The amino groups of amikacin play an important role in its antibacterial activity. One polymeric amikacin (denoted as amikacin (6)) has a stronger inhibitory effect on protein synthesis in vitro than other polymeric amikacins ( $8 \mathrm{a}$ and $8 \mathrm{~b}$ ) due to the modification of its side chains and amino groups [11]. Similarly, a recent study developed a polymeric vancomycin that has a much stronger antibiotic activity than monomeric vancomycin [12].

Targeting RNA for drug development is not a new concept. An antisense drug approach has been intensively investigated for decades, resulting in multiple clinical trials. Recently, high-field NMR analyses of the structures of RNA molecules, including Group 1 intron of hammerhead ribozyme [13, 14], HIV-1 mRNA Rev responsive element [15], and a trans-activation response element, revealed new structural motifs in a threedimensional manner $[16,17]$. These advances in elucidating the structures and functions of RNAs led the pharmaceutical industry to regard them as new therapeutic targets [18]. Because AGs can generally bind strongly to any type of RNA motifs, they may be plausible candidates for new RNA-targeting drugs.

In vitro selection of RNAs that bind to target molecules is usually performed over iterative cycles, in a process known as systemic evolution of ligands by exponential enrichment (SELEX) $[19,20]$. This method has enabled the identification of unique high affinity RNAs (aptamers) that bind to ligands of interest within large random RNA libraries [21, 22]. Aptamers are single-stranded short oligonucleotide or peptide molecules that bind to a specific target, including proteins, carbohydrates, and small molecules with high affinity and specificity [23, 24]. Since their initial discovery, numerous researchers have used aptamer selection as a means for widespread application and discovery of aptamers in diagnostic and therapy, as well as to approaches that could considerably expand the range of aptamer application [19]. Efforts to identify specific RNA sequences that bind to certain AGs will enhance our understanding of the rules underlying RNA-AG recognition, and will aid the development of sequence-specific RNA target therapeutics [24]. RNA aptamers against lividomycin have identical sequences to those of the Haemophilus influenza and Leishmania parasite rRNAs [25], suggesting that the sequences could be new RNA target sites used to control these pathogens. An aptamer against neomycin was also reported to have a certain stem loop structure with wobble non-Watson-Crick base pairings [26]. Lato et al. in 1995 reported six RNA aptamers (sla $21,26,110,150,151$, and 254) that recognized amikacin [27]. Recently, three DNA aptamers (Aptamer 15, 16, and 41) and one modified truncated aptamer (Aptamer 8-2) bind against streptomycin have been reported [28]. Moreover, an USA based world intellectual property organization published amikacin-binding five DNA aptamers [29]. The most successful RNA binding was achieved for neomycin B and tobramycin as compared to other AGs and the binding of these AGs antibiotics was at the region of the helix 69 of $23 S$ rRNA suggesting the site specific RNA binding of the AGs [30].

In the current study, we used SELEX to screen for RNA targets that bind to multivalent amikacin. After five cycles of selection, a characteristic sequence variation was observed among 38 clones. Overall, five groups of similar sequences (groups A, B, C, D, and E) were identified. The sequences of groups $\mathrm{A}$ and $\mathrm{B}$ were almost identical, suggesting that 
the selected RNA was enriched. Moreover, there was a significant sequence homology among different clones, and nine conserved motifs were identified. Subsequently, we used the Basic Local Alignment Search Tool (BLAST) program to search for similar motifs in bacterial rRNA sequences. Notably, we did not identifyany sequence homology to bacterial rRNAs, suggesting that the RNA motifs (aptamers) identified here may be new target sites for amikacin.

\section{Results and Discussion}

\subsection{Selection of RNA}

Small organic molecules are quite often challenging targets for an aptamer selection using SELEX method. Amikacin is such a molecule that belongs to the AG antibiotics having potential antibacterial activity [6]. To select amikacin-specific RNAs, multivalent amikacin was immobilized on epoxy-activated porous glass beads (genoglass PG-200EP) using ethanolamine under mild basic conditions [31]. Approximately $50 \%$ of the amikacin applied was immobilized on the porous glass beads suggests that the amikacin was successfully immobilized on the beads (Figure 1S). A linear N25 DNA template underwent polymerase chain reaction (PCR) amplification in the first selection cycle, and a total of five rounds of selection and amplification were performed. Figure 1 provides an overview of the RNA selection process. To monitor the progress of the selection process, the quantity of the single stand RNA (ssRNA) was measured by a UV spectrometer. As shown in the supplemental Figure 2S, the percentage binding affinity of ssRNA on amikacin-bound beads was in the range of $22-31 \%$ in the five selection rounds.

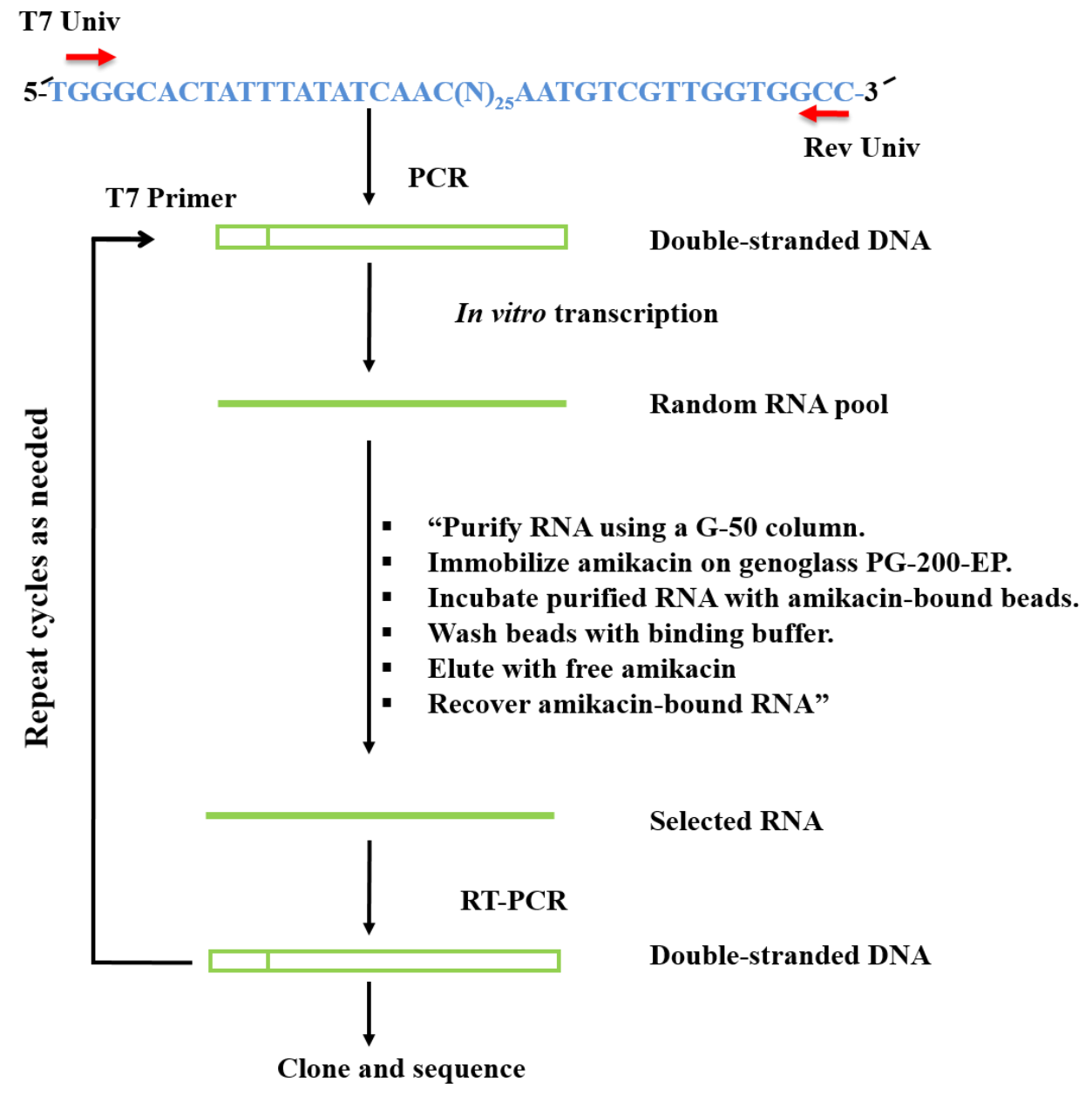


Figure 1. Strategy for RNA selection in vitro. The specific steps of selection and amplification are described in the Materials and Methods section. DNA and RNA are represented by double and single lines, respectively. The linear N25 template is shown, and the random nucleotides are represented by $(\mathrm{N}) 25$.

For the first selection cycle, seven rounds of PCR were performed, whereas 13 rounds of PCR were used for the subsequent selection cycles. Gel electrophoresis confirmed that a product of the expected size $(103 \mathrm{bp}$ ) was generated in each cycle (Figure 2).

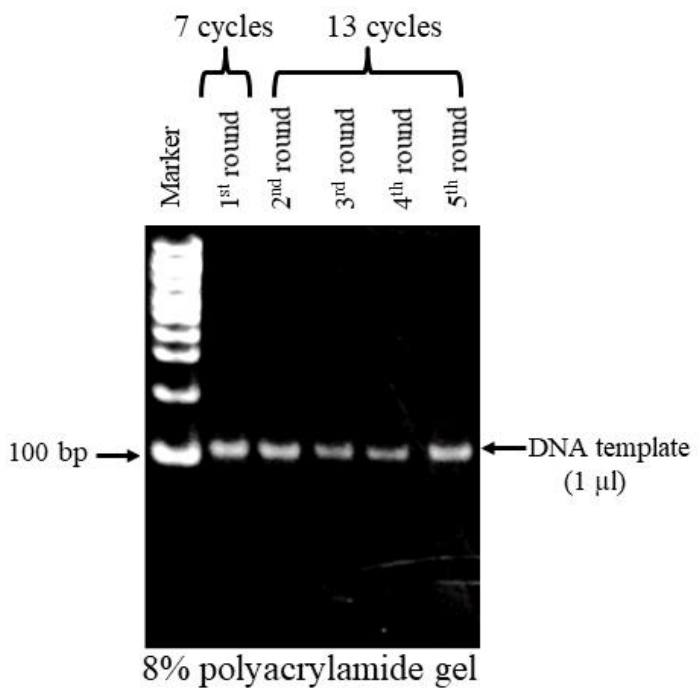

Figure 2. PCR results after five rounds of SELEX. After each cycle, the PCR mixture was run on an $8 \%$ polyacrylamide gel and stained with ethidium bromide. A 100 bp DNA marker was used. The expected product size was $103 \mathrm{bp}$.

In each cycle, the PCR product was digested with BamHI to generate a 97 bp product. The digested product was then purified using the phenol-chloroform extraction method and concentrated by ethanol precipitation. Aliquots $(1 \mu \mathrm{l})$ of each sample were run on an $8 \%$ polyacrylamide gel to confirm successful digestion and concentration of the 97 bp product (Figure 3). 


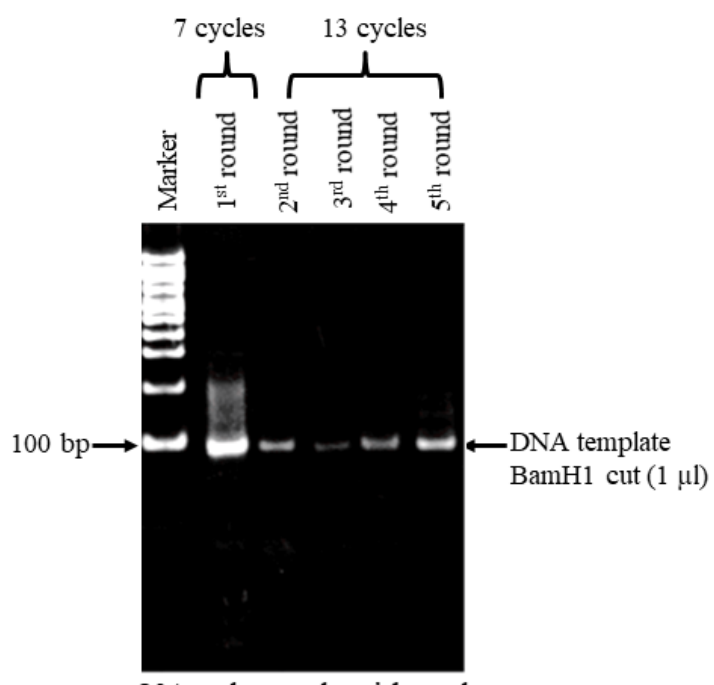

$8 \%$ polyacrylamide gel

Figure 3. DNA digested with BamHI. After each cycle, the digested DNA was run on an $8 \%$ polyacrylamide gel and stained with ethidium bromide. A 100 bp DNA marker was used. The expected product size was $97 \mathrm{bp}$.

Next, the digested and purified DNA was in vitro transcribed and the synthesized RNA was purified using a G-50 column. After each cycle, the purified RNA was run on an $8 \%$ polyacrylamide gel (Figure 4 ). The percentage of bound RNA was high in the early rounds and little improvement was observed among the five rounds. This might be because of complexity of RNA experiments in its many steps, and therefore not easy to receive constant absolute amounts of bound RNA in each round. Thus, a more detailed characterization of enrichment is needed. 


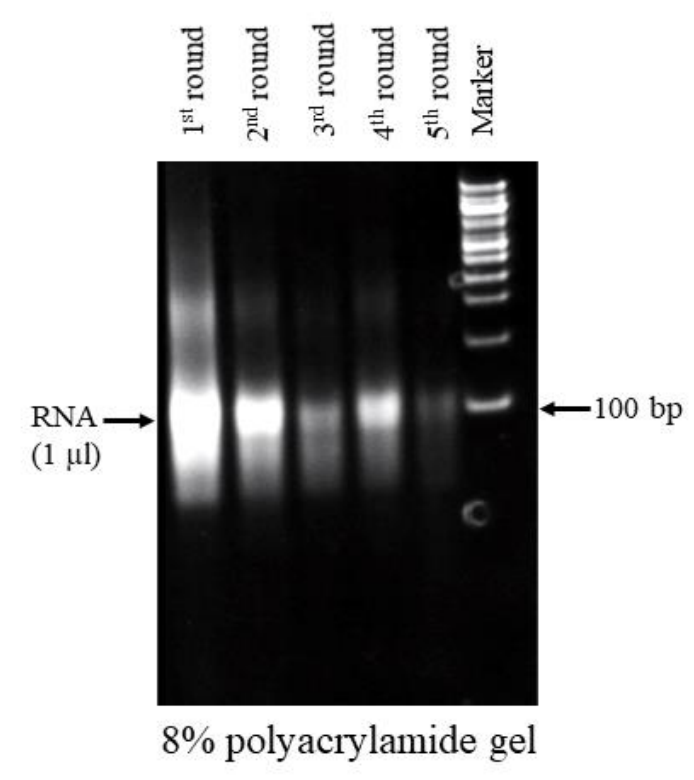

Figure 4. In vitro transcription after five rounds of SELEX. A 100 bp DNA marker was used.

Subsequently, the synthesized RNA was incubated with previously prepared amikacin-immobilized beads. Unbound RNAs and those that were weakly bound to the beads were removed by washing with binding buffer, and the remaining RNAs were eluted with free amikacin. The eluted RNAs were purified by ethanol precipitation, reverse transcribed, and then amplified by PCR for use in the subsequent cycles. The process was repeated for a total of five rounds. Overall, in this experiment, we obtained the expected size of DNA after PCR amplification and BamHI digestion, and synthesized RNA that bound to immobilized amikacin (Figure 2-4). Taken all the results together suggested the specificity and affinity of RNA aptamer to a small molecule like amikacin and would aid the development of sequence-specific RNA target therapeutics.

\subsection{Sequence analysis of amikacin-specific aptamers}

Since in vitro selection was introduced in the early 1990s [19], the power of this combinatorial approach has been used to generate aptamers that bind targets ranging from organic molecules to proteins and even DNA [32-34]. It is reported that more than 2000 aptamers have been synthesized during the past 30 years [22]. Accumulating evidence suggests that a large number of generated aptamers can bind various target molecules, including AGs [26-29]. In spite of such achievements, the successful clinical uses of these aptamers remain elusive. The U.S. Food and Drug Administration only approved the first aptamer-based drug, pegaptanib sodium (brand name: Macugen), for the treatment for age-related macular degeneration [35-36]. The major drawbacks of aptamer developments and clinical applications are time consuming and low successful rates. Moreover, the current aptamers are generated mostly by in vitro method and need to confirm their functions by in vivo study. Therefore, there is a lot of research scope to 
identify new aptamers using SELEX method that could be novel RNA specific therapeutic targets.

In this study, after five rounds of SELEX, the resulting PCR product was cloned into the pGEM-T-Easy vector and transformed into competent Escherichia coli cells. Figure 5 provides an overview of the method used to identify and analyze the sub-clones. Briefly, 50 clones were sub-cultured; among them, 38 clones were positive for the inserted region and were confirmed by sequencing. There were marked sequence variations among different clones, but five groups of similar sequences (groups A through E) were identified (Table 1). These results are alike to that of the data reported earlier [21, 25].

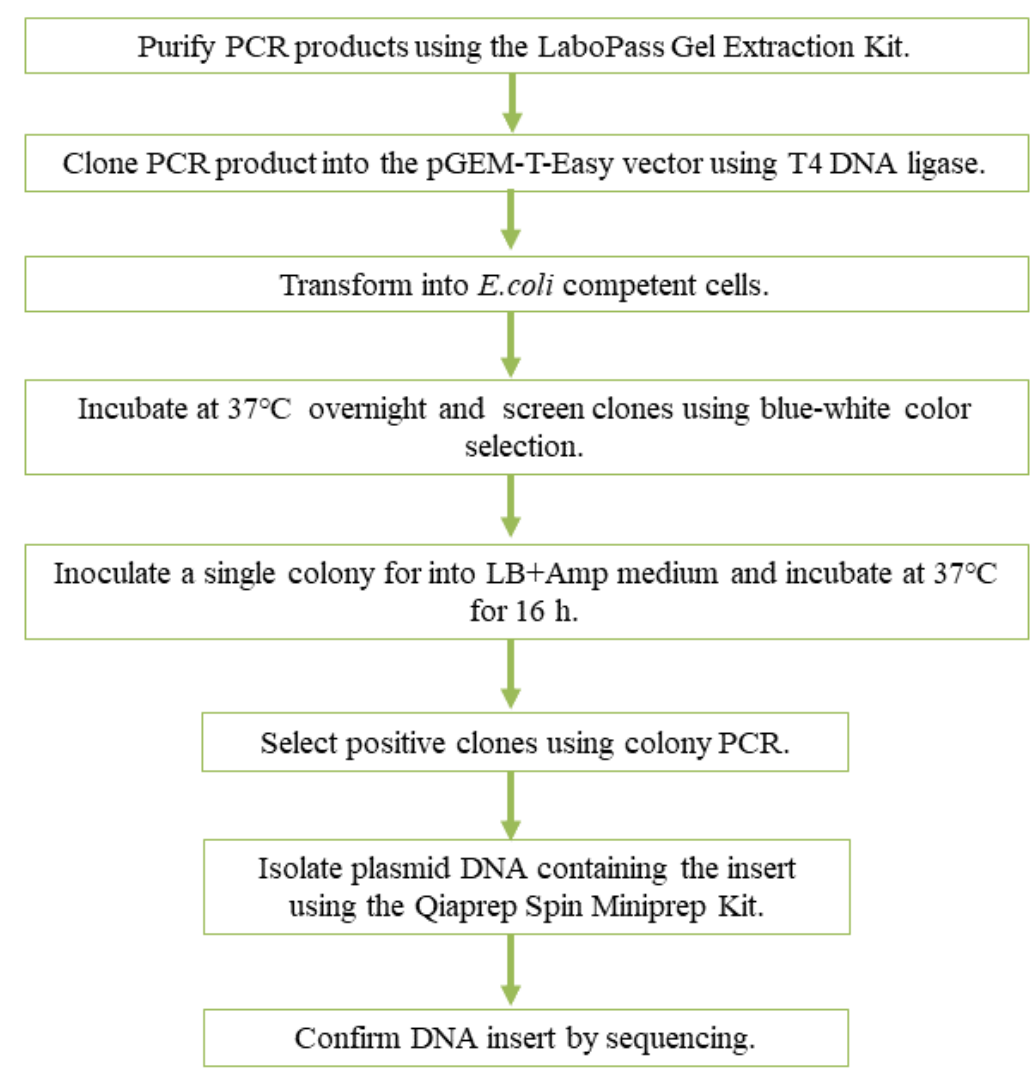

Figure 5. Overview of the methods used to identify and analyze sub-clones.

Table 1. Representative sequences of the five groups.

\begin{tabular}{ll}
\hline Group & Representative sequence \\
\hline GroupA & AGGUUAGCCGAUAAGAUCACCCUGA \\
\hline GroupB & UUAGCCGAUCAGUUCACgaugcuac \\
\hline GroupC & acgGCGGAGCCaaGAGCAGc \\
\hline GroupD & gAGACCCCCC(C)Ggy \\
\hline GroupE & yGACAGUCnUACUGACy \\
\hline
\end{tabular}

Lower-case letters indicate nucleotides that were not conserved among the clones. $\mathrm{Y}$ indicates a pyrimidine base.

From the five groups of sequences, nine conserved motifs were identified (Figure 6 and Table 2). The sequences in groups $\mathrm{A}$ and $\mathrm{B}$ were almost identical, suggesting that the selected RNA was enriched (Figure 6). Our data are consistent with those published previously $[19,20,22]$ where they reported that some of the identified sequences were more or less indistinguishable, indicates the enrichment of the selected RNA. 


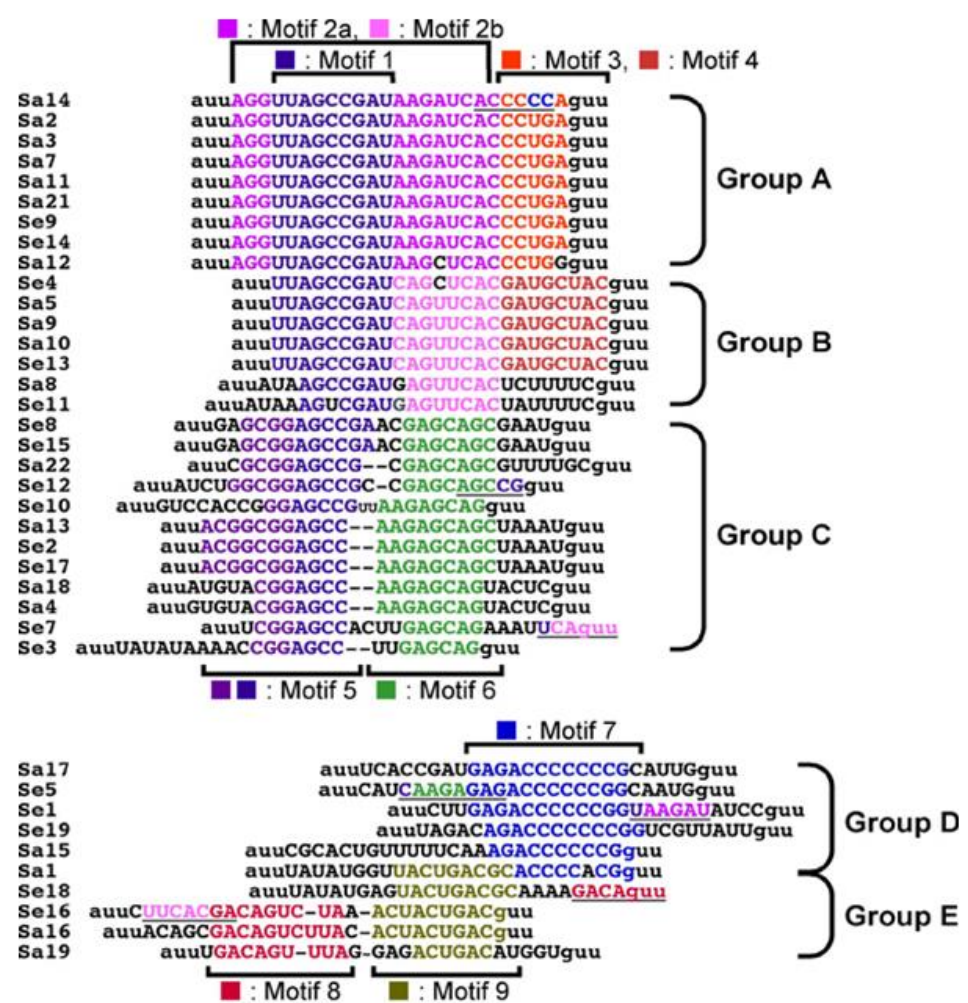

Figure 6. Nucleotide sequences of the 38 clones in groups A through E. The clone numbers are shown on the left-hand side. The sequences shown comprise only the 25 nucleotides that were random at the beginning of the selection. The sequences of the clones in groups A and B are almost identical. Nucleotides matching the consensus sequence are shown in different colors, whereas those shown in black differ from the consensus sequence.

Table 2. Motifs and their conserved sequences.

\begin{tabular}{ll}
\hline Motif & Consensus sequence \\
\hline Motif1 & UUAGCCGAU \\
\hline Motif 2a (include1) & AGG(UUAGCCGAU)AAGAUCAC \\
\hline Motif2b & CAGUUUCAC \\
\hline Motif3 & CCUGA \\
\hline Motif4 & GAUGCUAC \\
\hline Motif5 & acgGCGGAGCCra \\
\hline Motif6 & aaGAGCAGc \\
\hline Motif7 & gAGACCCCCC(C)Ggy \\
\hline Motif8 & yGACAGUCcua \\
\hline
\end{tabular}

Lower-case letters indicate nucleotides that are not consistent with the respective motif. $\mathrm{Y}$ indicates a pyrimidine base.

Amikacin is the most effective AG used to treat severe bacterial infections [4]. As mentioned above, amikacin inhibits protein synthesis and causes bacterial cell death by binding to $16 \mathrm{~S}$ rRNA [6]. Despite its activity against a number of bacterial species, resistance to amikacin can be achieved through several mechanisms; hence there is a need to identify new target binding sites of these AGs [9]. A number of studies described that both DNA and RNA aptamers are highly bound to amikacin [27, 29]. Recently, Soheili and her colleagues generated 3 aptames, which were significantly bound to streptomycin followed by amikacin [28]. It has been reported that neomycin-B specific aptamers (Neo1A and Neo2A) recognized 10 other AGs, including amikacin with similar affinities as for neomycin-B [37]. A kanamycin based DNA aptamer was selectively bound to the 
AG antibiotics, including amikacin [38]. Another study conducted by Ann et al, in 2010 reported effective RNA binding for neomycin B and tobramycin [30]. In the current study, we identified a number of unique conserved motifs (aptamers) that bind to amikacin. This result is consistent with the data published by Strehlitz et al. in 2012 [39], who showed aptamers as specific recognition elements for the detection of pharmaceuticals. Subsequently, we performed a BLAST search to identify bacterial 16S rRNA sequences containing the conserved motifs identified here. Strikingly, we did not find any sequence homology with bacterial $16 S$ rRNAs. These results suggest that amikacin has new binding sites that are not present in bacterial $16 \mathrm{~S}$ rRNAs. It has been reported previously that AGs conjugates are potential antiviral (anti-HIV) agents [40]. Moreover, antimicrobial activities of aptamers have been reported in a number of studies [41, 42]. Therefore, we suggest that the conserved motifs (aptamers) identified here not only could be novel target sites for amikacin but also could be used in targeted delivery mediated by these aptamers.

Future study is necessary to characterize the secondary structure of the identified aptamers and their binding, and affinity activities as well as specificity or selectivity against targets, such as other AG antibiotics. Aptamers that would show specificity to other aminoglycoside antibiotics, including amikacin could be used as therapeutical potentials as well as target for the elimination of those pharmaceutical or their residues from environment samples.

\section{Materials and Methods}

\subsection{Immobilization of amikacin on epoxy-activated porous glass}

The procedure for immobilization of amikacin has been described previously [31]. Briefly, amikacin solution $(1 \mathrm{mg} / \mathrm{ml})$ was prepared in $0.1 \mathrm{M} \mathrm{NaHCO}$ buffer $(\mathrm{pH} 9.0)$ and incubated with epoxy-activated porous glass beads (genoglass PG-200-EP) at room temperature (RT) overnight. The solution was then removed, and the glass beads were washed 5 times with buffer containing $0.1 \mathrm{M} \mathrm{NaHCO}_{3}(\mathrm{pH} 9.0)$ and $0.1 \mathrm{M}$ acetic acid $(\mathrm{pH}$ 4.0).After soaking with $1 \mathrm{M}$ ethanolamine at RT overnight, theporous glass beads containing amikacin were washed 9 times with water and used for SELEX. Spectrophotometrically at a wavelength of $524 \mathrm{~nm}$, the amount of immobilized amikacin on epoxyactivated porous glass was measured by subtracting the free amikacin obtained after washing from the amount of amikacin applied initially to the column. Later, the \% of immobilized amikacin on epoxy-activated porous glass was measured by dividing the immobilized amikacin on porous glass by the amount of amikacin applied initially to the column.

\section{2. $P C R$}

PCRs were carried out according to the method described by Alam et al.[43]. Briefly, the $50 \mu \mathrm{l}$ reactions contained $5 \mathrm{ng}$ of oligo DNA $1 \times$ Ex Taq Buffer, $0.2 \mathrm{mM} \mathrm{MgCl}, 2 \mu \mathrm{M}$ T7 primer, $2 \mu \mathrm{M}$ universal primer, and $0.5 \mathrm{U}$ of Ex Taq polymerase ( $1 \mathrm{U} / \mu \mathrm{l}$; TaKaRa). Thermal cycling was carried out in theGeneAmp PCR System 9700 under the following conditions:denaturation at $95^{\circ} \mathrm{C}$ for $3 \mathrm{~min}$, followed by seven cycles of $95^{\circ} \mathrm{C}$ for $30 \mathrm{~s}$, $54^{\circ} \mathrm{C}$ for 30 s, and $72^{\circ} \mathrm{C}$ for 30 s. A $1 \mu \mathrm{l}$ aliquot of each PCR sample was analyzedvia $8 \%$ polyacrylamide electrophoresis to confirm the size of the product. The remainder of each solution was concentrated by ethanol precipitation and resuspended in $10 \mu$ l of deionized water. Subsequently, a $9 \mu \mathrm{l}$ aliquot of the concentrated DNA was digested with 0.5 $\mathrm{U}$ of BamHI (TaKaRa) in $1 \times$ buffer $\mathrm{K}(20 \mu \mathrm{l}$ reaction volume $)$ at $37^{\circ} \mathrm{C}$ overnight. The digested sample was purified by phenol-chloroform extraction, concentrated by ethanol precipitation, and then dissolved in $10 \mu \mathrm{l}$ of deionized water.

\subsection{In vitro transcription}

In vitro transcription was performed according to the manufacturer's (Ambion) instructions. Briefly, the $10 \mu \mathrm{l}$ reaction included $1 \mu \mathrm{l}$ of digested and purified DNA, $1 \times \mathrm{T} 7$ 
MEGAscript buffer, $7.5 \mathrm{mM}$ each NTP, and $1 \mu \mathrm{l}$ of T7 MEGAshortscript enzyme. The solution was incubated at $37^{\circ} \mathrm{C}$ overnight. Subsequently, $1 \mathrm{U}$ of RNase free Turbo DNase ( $2 \mathrm{U} / \mu \mathrm{l}$; Ambion) was added and the mixture was incubatedat $37^{\circ} \mathrm{C}$ for $15 \mathrm{~min}$ to remove residual DNA. A $1 \mu \mathrm{l}$ aliquot of the resulting solution was analyzed by $8 \%$ polyacrylamide gel electrophoresis to detect the synthesized RNA. Next, a ProbeQuantG-50 micro-column (GE Healthcare) was equilibrated using equilibrium buffer (0.3 M Naacetate ( $\mathrm{pH}$ 5.2), $0.5 \mathrm{M}$ EDTA ( $\mathrm{pH} 8.0$ ), and 0.1\% SDS) and the synthesized RNA was added to the column along with $92 \mu \mathrm{l}$ of TEN buffer $(40 \mathrm{mMTris}-\mathrm{HCl}$ ( $\mathrm{pH} 7.5), 1 \mathrm{mM}$ EDTA ( $\mathrm{pH} 8.0$ ), and $150 \mathrm{mM} \mathrm{NaCl}$ ). The column wascentrifuged at 10,000 $\mathrm{g}$ for $2 \mathrm{~min}$ at RT, and the elute containing RNA $(100 \mu \mathrm{l})$ was collected. The solution was then purified by phenol-chloroform extraction and concentratedby ethanol precipitation. The concentration of the purified RNA was measured at $260 \mathrm{~nm}$ using a UV spectrophotometer.

\subsection{RNA purification and selection of RNA using a G-50 column}

RNA purification was performed as described previously [44]. A $50 \mu$ sample of amikacin-immobilized epoxy beads was put into a fresh $1.5 \mathrm{ml}$ tube and centrifuged at $10,000 \mathrm{~g}$ for $30 \mathrm{~s}$ at RT. The supernatant was removed, and $1 \mathrm{ml}$ of binding buffer (20 $\mathrm{mM}$ HEPES-KOH (pH 7.9), $200 \mathrm{mMKCl}, 5 \%$ glycerol, and $0.1 \%$ Triton X-100) was added to the tube. After centrifugation at $10,000 \mathrm{~g}$ for $30 \mathrm{~s}$, the supernatant was removed. Subsequently, $100 \mu \mathrm{l}$ of binding buffer, $1 \mu \mathrm{l}$ of RNasin ribonuclease inhibitor, and $1 \mu \mathrm{g}$ of in vitro transcribed RNA were added to a fresh $1.5 \mathrm{ml}$ tube and the tube was rotated for $1 \mathrm{~h}$ at $4^{\circ} \mathrm{C}$ and centrifuged at $10,000 \mathrm{~g}$ for $30 \mathrm{~s}$. The supernatant was removed, and the RNA was washed three times with $1 \mathrm{ml}$ of binding buffer and centrifuged at 10,000 $\mathrm{g}$ for $30 \mathrm{~s}$. For elution, $100 \mu \mathrm{l}$ of binding buffer, $1 \mu \mathrm{l}$ of RNasin, and $250 \mu \mathrm{g}$ of free amikacin were added and the tube was rotated at $4^{\circ} \mathrm{C}$ for $5 \mathrm{~min}$ following a quick centrifugation at $10,000 \mathrm{~g}$ for $30 \mathrm{~s}$. Subsequently, the solution was carefully transferred into a fresh $1.5 \mathrm{ml}$ tube containing $1 \mu \mathrm{l}$ of glycogen. The solution was then purified and concentrated (final volume: $10 \mu \mathrm{l}$ ) by phenol-chloroform extraction followed by ethanol precipitation. The concentration of amikacin-bound ssRNA was measured by subtracting the free ssRNA obtained after washing from the amount of ssRNA applied initially to the column at 260 $\mathrm{nm}$ using a UV spectrophotometer. Later, the \% of binding was measured by dividing the amikacin-bound ssRNA by the amount of ssRNA applied initially to the column.

\subsection{Reverse transcription}

The cDNA was prepared as described by Alam et al. [45]. Briefly, the $13 \mu$ l reaction contained $1 \mu \mathrm{l}$ of purified RNA,2 $\mu \mathrm{M}$ T7 universal reverse primer, and $0.5 \mathrm{mM}$ each dNTP. The solution was heated at $65^{\circ} \mathrm{C}$ for $5 \mathrm{~min}$ and then placed on ice for at least 1 min. Subsequently, $1 \times$ first strand buffer, $5 \mu \mathrm{M}$ DTT, 2 U of RNaseOUT, and $10 \mathrm{U}$ of SuperScript III reverse transcriptase (Invitrogen) were added to make the $20 \mu$ l final reaction volume. The reaction was incubated for $1 \mathrm{~h}$ at $50^{\circ} \mathrm{C}$, and theninactivated by heating at $70^{\circ} \mathrm{C}$ for $15 \mathrm{~min}$. The cDNA solution was then cooled, and a $1 \mu \mathrm{l}$ aliquot was used in $50 \mu \mathrm{l}$ PCR samples.

\subsection{In vitro selection (SELEX)}

In vitro selection experiments were carried out essentially as described by Abe et al. [46]. The starting RNA source was synthesized in vitro from a mixture of DNA templates containing a T7 promoter and a randomized 25 nucleotide region. The synthesized RNA was purified by adding $1 \mathrm{ml}$ of binding buffer (20 mM HEPES-KOH (pH 7.9), 200 $\mathrm{mMKCl}, 5 \%$ glycerol, and $0.1 \%$ Triton X-100) into a fresh tube. After centrifugation at $10,000 \mathrm{~g}$ for $30 \mathrm{~s}$, the supernatant was removed, and the RNA was washed three times with $1 \mathrm{ml}$ of binding buffer and centrifuged at 10,000 $\mathrm{g}$ for $30 \mathrm{~s}$. For elution, $100 \mu \mathrm{lof}$ binding buffer, $1 \mu \mathrm{l}$ of RNasin, and $250 \mu \mathrm{g}$ of free amikacin were added and the tube was rotated at $4^{\circ} \mathrm{C}$ for 5 min following a quick centrifugation at 10,000 g for $30 \mathrm{~s}$. Subsequently, the solution was carefully transferred into a fresh $1.5 \mathrm{ml}$ tube containing $1 \mu \mathrm{l}$ of 
glycogen. The solution was then purified and concentrated (final volume: $10 \mu \mathrm{l}$ ) by phenol-chloroform extraction followed by ethanol precipitation and selected using a G-50 column. Subsequently, the selected RNA was converted to single-stranded cDNA, which was amplified by PCR and then transcribed in vitro and used for the next round of selection. Five rounds of selection and amplification were performed (Figure 1).

\subsection{Cloning and sequencing}

In vitro cloning and sequencing were performed as described by Alam et al. [45]. The PCR products were purified using the LaboPass Gel Extraction Kit (Cosmogenetech), followed by phenol-chloroform extraction. The purified PCR products were inserted into the pGEM-T-Easy vector (Promega). The plasmid DNA containing the insert was purified using the QIAprep Spin Miniprep Kit (Promega) and sequenced using M13 forward primers and the BigDyeTerminator v3.1 Cycle Sequencing Kit (Applied Biosystems). Sequences were determined using an ABI Prism 3100 Genetic Analyzer (Applied Biosystems) and confirmed using BLAST software.

Author Contributions: T.T. and Y.M. designed and supervised the work. Both T.T. and Y.M. have contributed to, seen and approved the manuscript. AHM.K.A. carried out the experiments and prepared the manuscript. All authors read the manuscript and approved it for submission.

Funding: This work was supported in part by grants-in-aid from the Japan Society for the promotion of Science (17H02204 and 18K19288).

Data Availability Statement: All data are available within the manuscript.

Acknowledgments: We gratefully acknowledge scholarships from Ministry of Education, Culture, Sports, Science and Technology (MEXT), Japan.

Conflicts of Interest: The authors declare no conflict of interest.

\section{References}

1. Liu, Q.; Li, J.; Song, X.; Zhang, M.; Li, E.; Gaob, F.; He L. Simultaneous determination of aminoglycoside antibiotics in feeds using high performance liquid chromatography with evaporative light scattering detection. RSC Adv. 2017, 7, 1251-1259.

2. Luan, $\mathrm{Y} ;$ Wang, N; Li, C; Guo, $\mathrm{X} ; \mathrm{Lu}, \mathrm{A}$. Advances in the application of aptamer biosensors to the detection of aminoglycosides antibiotics. Antibiotics, 2020, 9, 787.

3. Kevin, M.K.; Alisa, W.S.; Timothy, R.K.; Lynn, E.C. Aminoglycosides: An Overview. Cold Spring Harb. Perspect. Med. 2016, 6, a027029.

4. Smritilekha, B.; George, G.Z.; Frank, S. Antibacterial activities of aminoglycoside antibiotics-derived cationic amphiphiles. Polyol-modified Neomycin B-, Kanamycin A-, Amikacin-, and Neamine-based amphiphiles with potent broad spectrum antibacterial activity. J. Med. Chem. 2010, 53, 3626-3631.

5. Lambert, M.; Bruyndonckx, R.; Goossens, H.; Hens, N.; Aerts, M.; Catry, B.; Neely, F.; Vogelaers, D.; Hammami. N. The Belgian policy of funding antimicrobial stewardship in hospitals and trends of selected quality indicators for antimicrobial use, 1999-2010: a longitudinal study. BMJ Open 2015, 5, e006916.

6. Kotra, L.P.; Haddad, J.; Mobashery, S. Aminoglycoside: perspectives on mechanisms of action and resistance and strategies to counter resistance. Antimicrob. Agents Chemother. 2000, 44, 3249-3256.

7. Ramirez, M.S.; Tolmasky, M.E. Aminoglycoside modifying enzymes. Drug Resist. Updat. 2010, 13, 151-171.

8. Peloquin, C.A.; Berning, S.E.; Nitta, A.T.; Simone, P.M.; Goble, M.; Huitt, G.A.; Iseman, M.D.; Cook, J.L.; Curran-Everett, D. Aminoglycoside toxicity: daily versus thrice-weekly dosing for treatment of mycobacterial diseases. Clin. Infect. Dis. 2004, 38, 1538-1544.

9. $\quad$ Edson, R.S.; Terrell, C.L. The aminoglycosides. Mayo. Clin. Proc. 1999, 74, 519-28.

10. Poole, K. Efflux-mediated antimicrobial resistance. J. Antimicrob. Chemother. 2005, 56, 20-51.

11. Miura, Y. I.; Wada, N.; Kobayashi, K. Chemoenzymatic synthesis of a multivalent aminoglycoside. Macromol. Biosci. 2003, 3, 662-667.

12. Ashraf, Z.; Heather-Rose, M.; Dimitri, Y.C.; Andrew, D. B.; Andrew, R.; Hesketh, H. H. Zn(II) mediates vancomycin polymerization and potentiates its antibiotic activity against resistant bacteria. Sci. Rep. 2017, 7:4893.

13. Stage, T.K.; Hertei, K.J.; Uhlenbech, O.C. Inhibition of hammerhead ribozyme by neomycin. RNA 1995, 1, 95-101. 
14. Ahsen, U.; Davies, J.; Schroder, R. Antibiotic inhibition of group 1 intron ribozyme function. Nature 1991, 353, 368-370.

15. Zapp, M.L.; Stern, S.; Green, M.R. Small molecules that selectively block RNA binding of HIV-1 Rev protein inhibit Rev function and viral production. Cell 1993, 74, 969-978.

16. Hendrics, M.; Priestley, E.S.; Joyce, G.F.; Wong, C-H. Direct observation of aminoglycoside-RNA interactions by surface plasmon resonance. J. Am. Chem. Soc. 1997,119, 3641-3648.

17. Mei, H.N.; Galan, A.A.; Halim, N.S.; Mack, D.P.; Moreland, D.W.; Sanders, K.B.; Truong, H.N.; Czernik, A.W. Inhibition of an HIV-1 Tat derived peptide binding to TAR RAA by aminoglycoside antibiotics. Bioorg. Med. Chem.Lett. 1995, 5, $2755-2760$.

18. Noreen, F.R.; Graham, F.S. RNA as a small molecule druggable target. Bioorg. Med. Chem. Lett. 2017, $27,5083-5088$.

19. Ellington, A.D.; Szostak, J.W. In vitro selection of RNA molecules that bind specific ligands. Nature 1990, 346, 818-822.

20. Tuerk, C.; Gold, L. "Systematic evolution of ligands by exponential enrichment: RNA ligands to bacteriophage T4 DNA polymerase." Science 1990, 249, 505-510.

21. Liu, Q; Zhang, W; Chen, S; Zhuang, Z; Zhang, Y; Jiang, L; Sheng, J. SELEX tool: a novel and convenient gel based diffusion method for monitoring of aptamer-target binding. J. Biol. Eng., 2020, 14,1.

22. Yue, F.; Li, F.; ong, Q.; Guo, Y.;Sun, X. Recent advances in aptamer-based sensors for aminoglycoside antibiotics detection and their applications. Sci. Tot. Envir., 2021, 762,

23. Zhuo, Z.; Yu, Y.; Wang, M.; Li, J.; Zhang, Z.; Liu, J.; Wu, X.; Lu, A.; Zhang, G.; Zhang, B. Recent advances in SELEX technology and aptamer applications in biomedicine. Int. J. Mol. Sci. 2017, 18, 2142.

24. Huang, J.; Chen, X.; Fu, X.; Li, Z.; Huang, Y.; Liang, C. Advances in aptamer-based biomarker discovery.Front. Cell Dev. Biol. 2021,9,659760.

25. Lato, S.M.; Ellington, A.D. Screening chemical libraries for nucleic-acid-binding drugs by in vitro selection: a test case with lividomycin. Mol. Divers.1996, 2, 103-110.

26. Julia, E.; Weigand, M.S.; Ewald-bernd, U.; Sabrina, Z.; Renee, S.; Beatrix, S. Screening for engineered neomycin riboswitches that control translation initiation. RNA 2008, 14, 89-97.

27. Lato, S.M.; Boles, A.R.; Ellington, A.D. In vitro selection of RNA lectins: using combinatorial chemistry to interpret ribozyme evolution. Chem. Biol.1995, 2, 291-303.

28. Soheili,V.; Taghdisi, S.M.; Khayyat, M.H.;Bazzaz, B.S.F.;Ramezan, M.; Abnous, K. Colorimetric and ratiometric aggregation assay for streptomycin using gold nanoparticles and a new and highly specific aptamer. Microchim. Acta 2016, 183, 16871697.

29. World Intellectual Property Organization. Amikacin-binding primary aptamers, 2017, (PCT) WO 2017/210683 Al, 75-79.

30. Ann, E.S.; William, D.G.; Vendeix, FA.; Paul, F.A. Binding of aminoglycoside antibiotics to helix 69 of $23 \mathrm{~S}$ rRNA. Nuc. Acids Res. 2010, 38, 3094-3105.

31. Abood, L.G.; Latham, W.; Grassi, S. Isolation of a nicotine binding site from rat brain by affinity chromatography. Proc. Natl. Acad. Sci. 1983, 11, 3536-3539.

32. Beaudry, A.; Joyce, G.F. Directed evolution of an RNA enzyme. Science 1992, 257, 635-64.

33. Gold, L.; Polisky, B.; Uhlenbeck, O.; Yarsus, M. Diversity of oligonucleotide functions. Annu. Rev. Biochem. $1995,64,763-797$.

34. Pei, D.; Ulrich, H.D.; Schultz, P.G. A combinatorial approach toward DNA recognition. Science 1991, 253, 1408-1411.

35. Siddiqui, M.A.A.; Keating, G.M. Pegaptanib:in exudative age-related macular degeneration. Drugs 2005, 65, $1571-1577$.

36. Dombi,T.; Kwok, K.K.; Sultan, M.B. A retrospective, pooled data analysis of the safety of pegaptanib sodium in the treatment of age-related macular degeneration in subjects with or without diabetes mellitus. BMC Ophthalmol. 2012, 12: 37.

37. Ilgu, M; Fulton, D.B.; Yennamalli, R.M.; Lamm, M.H.; Sen, T; Nilsen-Hamilton, M. An adaptable pentaloop defines a robust neomycin-B RNA aptamer with conditional ligand-bound strictures. RNA 2014, 20, 1-10.

38. Yunfei, T; Gu, C; Wang, C; Song, B; Zhou, X; Lou, X; He, M. Evanescent wave aptasensor for continious and online aminoglycoside antibiotics detection based on target binding facilitated fluorescence quencing. Bios. Bioele. 2018, 102, 646-651.

39. Strehlitz, B.; Reinemann, C.; Linkorn, S.; Stoltenburg, R. Aptamers for pharmaceuticals and their application in environmental analytics. Bioanal. Rev. 2012, 4, 1-30.

40. Cabrera, C.; Gutierrez, A.; Blanco, J.; Barretina, J.; Litovchick, A.; Lapidot, A.; Evdokimov, A.G.; Clotet, B.; Este, J.A. Antihuman immunodeficiency virus activity of novel aminoglycoside-arginine conjugates at early stages of infection. AIDS Res. Human Retrov. 2000, 16, 627-634. 
41. Afrasiabi, S; Pourhajibagher, M; Raoofian, R; Tabarzad, M; Bahadur, A. Therapeutic applications of nucleic acid aptamers in microbial infections. J Biomed Sci. 2020, 27, 6.

42. Zou, X; Wu, J; Gu, J; Shen, L; Mao, L. Application of aptamers in virus detection and antiviral therapy. Front. Microbial. 2019, 10,1462 .

43. Alam, A.H.M.K.; Hossain, A.S.M.S.; Khan, M.A.; Kabir, S.R.; Reza, M.A.; Rahman, M.M.; Islam, M.S.; Rahman, M.A.A.R.; Rashid, M.; Sadik, MG. The antioxidative fraction of white mulberry induces apoptosis through regulation of p53 and NFkB in EAC cells. PLoS ONE 2016, 11, e0167536.

44. Alam, A.H.; Suzuki, H.; Tsukahara, T. Expression analysis of Fgf8a \&Fgf8b in early stage of P19 cells during neural differentiation. Cell Biol. Int. 2009, 33,1032-1037.

45. Alam, A.H.; Suzuki, H.; Tsukahara, T. Retinoic acid treatment and cell aggregati.on independently regulate alternative splicing in P19 cells during neural differentiation. Cell Biol. Int. 2010, 34, 631-643.

46. Abe, R.; Sakashita, E.; Yamamoto, K; Sakamoto, H. Two different RNA binding activities for the AU-rich element and the poly(A) sequence of the mouse neuronal protein mHuC. Nuc. Acids Res. 1996, 24, 4895-4901.

\section{Supplemental Data}

\section{Figure Legend}

Figure1S. Immobilization of amikacin on epoxy-activated porous glass measured after each round. \% immobilization $=$ (the amount of the immobilized amikacin on the bed)/(the amount of the applied amikacin to the column)x 100.

Figure 2S. Enrichment profile of the ssRNA pool measured the \% binding after each round. \% binding $=($ the amount of the bound ssRNAs)/(the amount of the applied ssRNAs to the column)x 100. 